\section{„Wir laden sie schon jetzt herzlich ein, mit einer Vortrags- oder Poster- anmeldung aktiv beizutragen."}

Prof. Dr. Carl-Peter Bauer, Fachklinik Gaißach Prof. Dr. Dr. Johannes Ring, Klinik und Poliklinik für Dermatologie und Allergologie am Biederstein, Technische Universität München

\title{
Allergien von der Schwangerschaft bis ins Senium
}

V om 11. bis zum 13. Oktober 2012 findet in München der 7. Deutsche Allergiekongress statt. Dazu laden wir Sie herzlich ein.

Veranstaltet wird dieser Kongress von den drei Fachgesellschaften GPA, DGAKI und ÄDA und als Hauptthema haben wir „Allergien in verschiedenen Lebensabschnitten“ gewählt. Beginnen werden wir am ersten Tag mit Schwangerschaft, Neugeborenenzeit und früher Kindheit. Daran schließt sich das Erwachsenenalter mit dem Berufsleben an und am

\section{Deutscher Allergiekongress auf einen Blick}

\section{Veranstalter}

_Ärzteverband Deutscher Allergologen (ÄDA)

_Deutsche Gesellschaft für Allergologie und klinische Immunologie (DGAKI)

_Gesellschaft für Pädiatrische Allergologie und Umweltmedizin (GPA)

\section{Kongresstermin und -ort}

11.-13. Oktober 2012

Forschungszentrum der Technischen Universität München, Garching

\section{Kongresspräsidenten}

_Prof. Dr. Carl-Peter Bauer, Gaißach

_Prof. Dr. Dr. Johannes Ring, München

\section{Anmeldung und Information}

Aktuelle Informationen im Internet unter www.allergiekongress.de, Anmeldung mit nebenstehendem Formular oder ebenfalls online dritten Kongresstag möchten wir die Allergieprobleme im Senium besprechen.

Ein besonderes Anliegen bei diesem Kongress ist uns auch die Diskussion über die aktuellen Forschungsergebnisse in der Allergologie. Wir laden sie schon jetzt herzlich ein, dazu mit einer Vortrags- oder Posteranmeldung aktiv beizutragen.

Einen weiteren Schwerpunkt wird wie in den Vorjahren die praktische Fortbildung in Kleingruppen mit interaktiven Seminaren und Workshops darstellen. In Ergänzung dazu werden wir erstmals für Medizinische Fachangestellte einen Fortbildungskurs durchführen und hoffen hier auf reges Interesse.

Das Symposium mit den Veterinärmedizinern über Allergien bei Tieren wird ebenfalls einen neuen Programmteil bilden und wir erwarten einen regen interdisziplinären Meinungs- und Erfahrungsaustausch.

Tagen werden wir vor den Toren von München in Garching auf dem neuen Campus der Technischen Universität, der sowohl mit der U-Bahn (U6) als auch mit dem PKW gut zu erreichen ist.

Neben der Wissenschaft gehört aber auch der abendliche kollegiale Meinungsaustausch zu unserem Programm, und wir möchten Sie bereits heute zu den Abendveranstaltungen in das Zentrum von München einladen.

Seien Sie herzlich willkommen! Wir freuen uns, Sie zahlreich in München begrüßen zu dürfen.
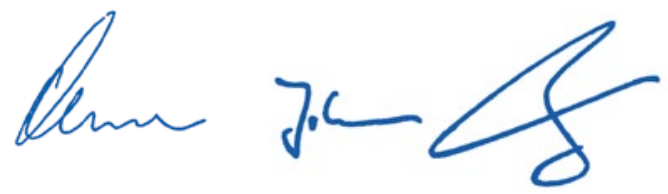